\title{
un proyecto autónomo es posible PARA ALUMNOS CON N.E.E.
}

\author{
Antonio Nieva Martínez, Teresa Fernández Morodo \\ e Isabel Desmonts Bengoechea \\ Orientadores educativos y terapeutas de familia y de pareja \\ idesmonts@hotmail.com
}

Añadiendo el área
afectivo-social en el currí-
culum de los alumnos con
N.E.E. les ampliamos la
posibilidad de disfrutar de
las ventajas de la inclusión
en centros ordinarios.
Éste área ha de diseñarse
individualmente valorando
las características per-
sonales y momento evo-
lutivo de estos alumnos.
Así pues, logra un papel
primordial el ámbito de
las habilidades sociales
dentro de éste área para
los alumnos de ESO.

I beneficio que obtiene un alumno con discapacidad de su inclusión en un centro ordinario es la posibilidad de crecer y madurar teniendo como modelo los patrones comunes de comportamiento, relación y aprendizaje del conjunto de los niños y niñas de su edad.

Para ello el niño tiene que llegar a estar en condiciones de interaccionar con los compañeros y con el entorno para lo cual son necesarios unos requisitos previos: un aceptable grado de autoestima, de confianza en sí mismo, capacidad de relacionarse con los demás niños y con los adultos y capacidad de desenvolverse e interaccionar con el entorno.

Este es el motivo por el que al iniciar el programa de atención a alumnos con necesidades educativas especiales (N.E.E.) en el año 1986, junto con el resto de las áreas del currículum ordinario, incluimos de manera específica el área afectivo social.

Con el paso del tiempo este área ha ido adquiriendo un carácter fundamental que nos permite desarrollar en nuestros alumnos con discapacidad los tres grandes ámbitos que hemos mencionado: la relación consigo mismo, la relación con los iguales y la relación con el entorno: aprendizaje, desenvolvimiento y autonomía.

Estos tres puntos constituyen los ejes que orientan la programación curricular de este área a lo largo de toda la escolaridad, tanto en lo que se refiere al establecimiento de los objetivos y contenidos como a la evaluación.

En cada etapa, como es lógico, los objetivos que se programan son diferentes, están adaptados a las características y necesidades de la edad y, progresivamente, se van haciendo más complejos a medida que los niños los van asimilando. De forma paralela, estos objetivos se van adaptando a las circunstancias individuales de cada alumno.

Esta idea nos permite estar al tanto y atender las necesidades personales y sociales que cada uno de nuestros alumnos presentan cuando inician su escolaridad en un centro inclusivo como el nuestro y programar las habilidades o estrategias de las que carecen o que no dominan de forma que su adaptación a la

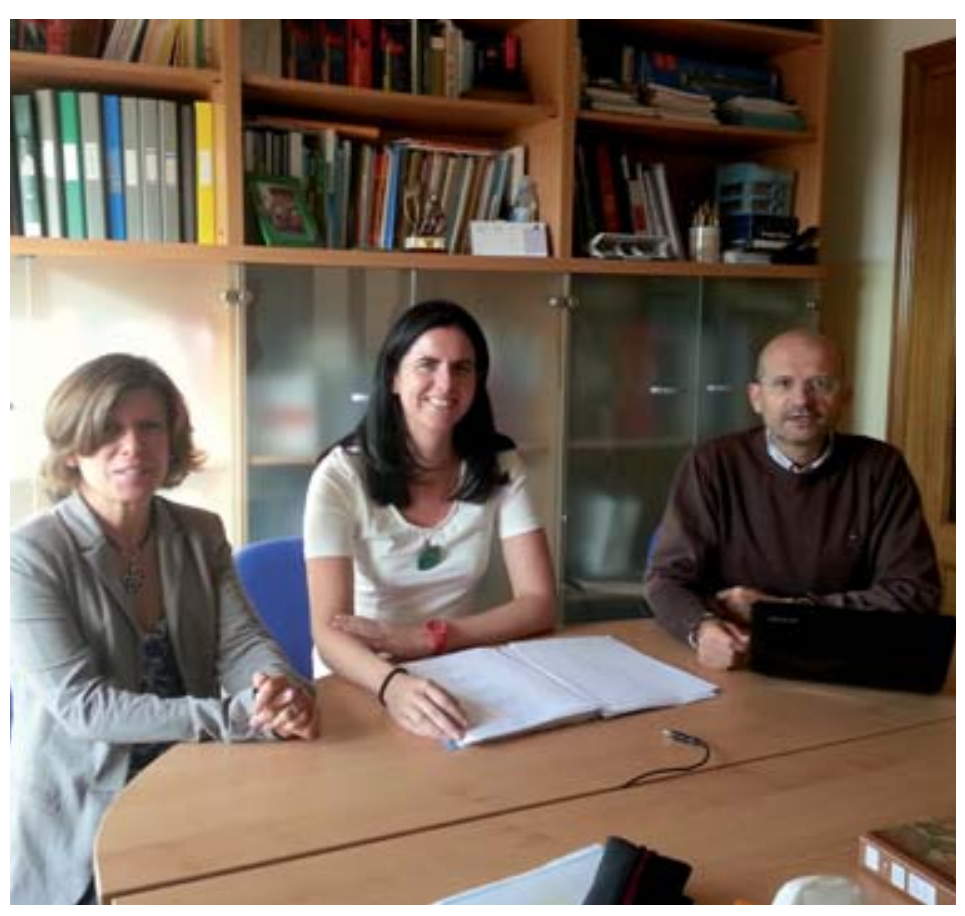

Antonio Nieva, Teresa Fernández e Isabel Desmonts. 
dinámica del centro y de la etapa, la comunicación con los demás y la relación con los iguales sea cada vez más ajustada y les permita una verdadera integración escolar y social.

En la etapa, de Educación Infantil centramos nuestra atención en que los niños se adapten al colegio, se encuentren a gusto en sus clases y puedan ir aprendiendo, de forma progresiva, como lo hace el resto de sus compañeros, las rutinas de funcionamiento propias de la etapa.

Los objetivos generales para esta etapa son:

- Adaptación al colegio y a la dinámica de funcionamiento de la etapa.

- Confianza en sí mismo.

- Relación con el adulto como figura de referencia y ayuda.

- Relación con los compañeros.

- Relación con el entorno hábitos, normas y rutinas.

En esta etapa la interacción que se establece entre todos los niños es un factor de motivación muy importante para los alumnos N.E.E. dada la actitud activa y abierta de los niños a esta edad.

En la etapa, de Educación Primaria nos vamos proponiendo nuevas metas que se traducen en objetivos más exigentes:

I. Que sepan diferenciar los momentos de juego y los momentos de trabajo.

2. Que sean capaces de tolerar la frustración.

3. Que incorporen a otros profesores y adultos como figuras de referencia y autoridad.

4. Que sean capaces de mantenerse en una actividad de juego o trabajo sin molestar a los compañeros.

5. Que sean capaces de ponerse en el lugar de los demás y les respeten.

6. Que respeten las normas de convivencia de su clase, que aprendan a resolver los conflictos sin recurrir al contacto físico ni al insulto, que vayan ajustando su comportamiento a las distintas ocasiones o dinámicas del aula.

El acceso a la etapa de secundaria de los alumnos N.E.E. en los institutos y colegios ha presentado especiales dificultades de modo que muchos centros llegaban a desistir y a plantear como solución la alternativa de derivar a los alumnos, en este tramo educativo, a Educación Especial.

La problemática que se plantea y confiere a la ESO unas características específicas a la hora de establecer la continuidad de la inclusión de alumnos con discapacidad se deriva de tres motivos: los cambios que experimentan todos los alumnos en general y los alumnos N.E.E. en concreto, las características particulares de la ESO y las expectativas y deseos no cumplidos de los padres.

\section{Cambios personales de los alumnos}

Al final de la etapa de Educación Primaria los alumnos con discapacidad acceden, unos antes y otros después, pero como el resto de sus compañeros, al periodo de la pubertad por lo que se ven sumidos en el lógico proceso de reorganización personal y social.
Muchos de los aspectos de su identidad que habían ido desarrollando y consolidando en la fase anterior se ponen en cuestión. Los chicos y las chicas empiezan a ser mucho más conscientes de sus necesidades y carencias y las soportan mal; manifiestan malestar al percibir los avances de sus compañeros de aula, quieren copiar sus actitudes, sus intereses, el modo de vestir o cómo se relacionan entre ellos y, por consiguiente, intentan imitarles pero no consiguen el éxito social que ven en ellos.

Como forma de protegerse tienden a juntarse por afinidades, gustos y edad mental, es el intento de sentirse comprendidos y aceptados. Esto les lleva a separarse de forma más evidente de sus compañeros de aula, que hasta entonces habían sido un elemento de apoyo e integración muy importantes.

Por un lado les cuesta entender lo que les está pasando personalmente y por otro tienen dificultades para entenderse con sus amigos y caen constantemente en discusiones que no aciertan a resolver.

Igualmente, los compañeros sin discapacidad acceden también al mismo proceso de desestructuración y reorganización. Manifiestan los mismos síntomas de cuestionamiento de su identidad, de necesidad de refugio en el grupo de los afines y de crítica y rechazo hacia lo que sienten como diferente y que, por lo tanto, les supone un cuestionamiento.

Nos encontramos así con una población de chi$\cos$ y chicas con tendencia a la rigidez, que utilizan como elemento de comunicación la ironía y la crítica, en búsqueda de su identidad y de su estabilidad y con el mecanismo del rechazo hacia todo lo que puedan vivir como amenaza hacia ellos y su proceso de asentamiento.

A los alumnos de enseñanza ordinaria se les pide que traten a sus compañeros con discapacidad eludiendo las actitudes infantiles o de sobreprotección. Por lo tanto les someten a las mismas reglas por las que se rigen entre ellos y emplean la ironía, la burla, la crítica y esperan que sepan entender este lenguaje y esta forma de relación. Los chicos con discapacidad intelectual no comprenden esta dinámica y se sienten rechazados y menospreciados.

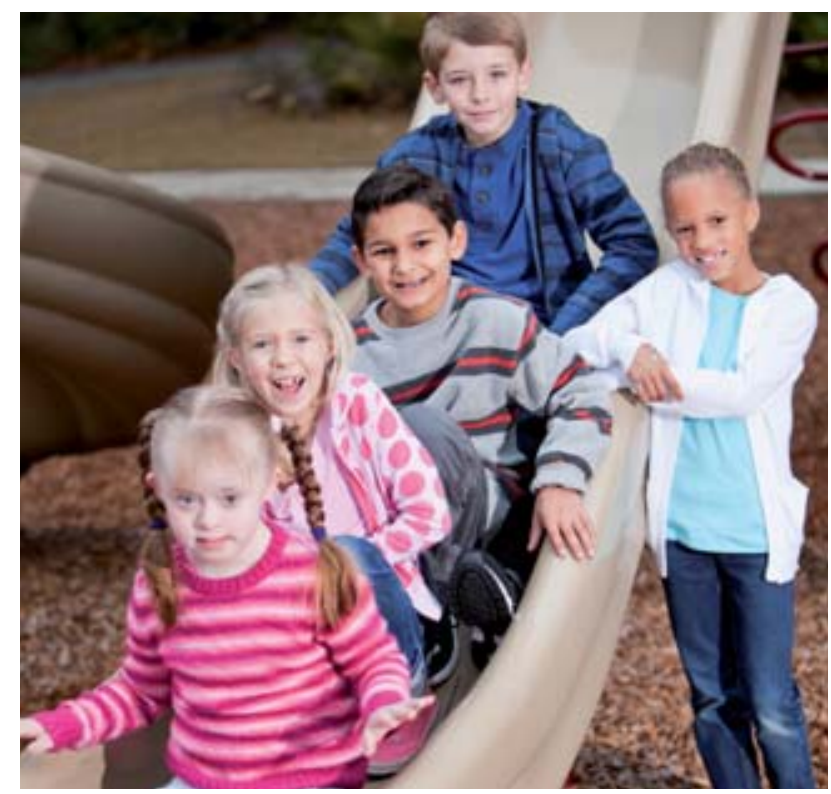


Esta actividad es un cuestionario de evaluación de principio y de final de uno o varios cursos de habilidades sociales que pueden utilizar los profesores.

\section{Cuestionario PARA PROFESORES}

I. Presto atención (mirando a los ojos, o al rostro) a quien me está hablando.

2. Pregunto enseguida cuando no entiendo algo (a los profesores, adultos, amigos) o cuando me resulta complicado.

3. No tengo dificultad para integrarme en un grupo o en una actividad.

4. Disfruto entablando conversación con conocidos y extraños.

5. Soy abierto y franco en lo que respecta a mis sentimientos.

6. Si alguien se me cuela en una fila, le llamo abiertamente la atención.
7. Expreso ante la gente mis opiniones.

8. He evitado hacer preguntas por miedo a parecer tonto.

9. Con frecuencia no sé qué decir a personas del otro sexo.

10. Defiendo mis derechos y opiniones dando a conocer cuál es mi postura sobre el tema.

II. Expreso mi malestar cuando un amigo me ha hecho algo que no me ha gustado.

12. Si observo que un grupo de compañeros me deja solo, pido que me incluyan.

13. Decido lo que quiero hacer cuando los demás (el grupo de amigos) quieren que haga otra cosa distinta y que sé que no me interesa realizar.

14. Resuelvo la sensación de aburrimiento en el grupo, proponiendo actividades interesantes.

15. Llamo la atención a alguien que dice o hace algo que me molesta íntimamente.
En este escenario no es difícil que la interacción entre alumnos con y sin discapacidad se torne más complicada que en edades anteriores. No es nada fácil la tarea a la que se enfrentan unos y otros.

\section{Condicionantes de LA ESO}

Junto con las cambiantes circunstancias personales hay que contar con las características de la etapa de Educación Secundaria. Tanto su estructura como el currículum en general son una fuente de obstáculos que es preciso considerar. De la referencia tan importante que supone la figura del tutor en primaria, tanto en el ámbito académico como en el personal en esta etapa los alumnos deben incorporar un profesor por asignatura, cada uno de ellos con su propio estilo personal de enseñanza y organización. Además, la presencia del tutor en el grupo de clase disminuye, por lo que no es posible contar con él de forma tan continuada e inmediata como antes.

Por otra parte las áreas del currículum presentan un grado de complejidad y abstracción que hacen muy difícil y en muchos casos imposible una mínima adaptación curricular accesible para los alumnos con discapacidad intelectual, por lo que su presencia en el aula ordinaria disminuye y se hace para ellos más costosa.

\section{LAS EXPECTATIVAS Y DECEPCIONES DE LOS PADRES}

El apoyo y la colaboración de los padres de los alumnos N.E.E. es un factor determinante. Cuando los chicos y chicas con discapacidad acceden a la ESO los padres también perciben y experimentan los cambios que acabamos de explicar. $Y$ ante los aspectos que aparentemente pierden: sus hijos dejan de ser unos niños ya no les necesitan tanto, ya no obedecen como antes, la relación con los compañeros del aula es menor, el tiempo de permanencia en las aulas se reduce, etcétera, es muy frecuente que aparezcan sentimientos de decepción y malestar que les desaniman y que hacen que se muestren descontentos y críticos con el proceso de inclusión que lleva al cabo el centro.
Se pasa por momentos en los que, debido a este malestar, la colaboración de los padres con el colegio puede alterarse con el consiguiente perjuicio en la necesaria labor complementaria que debe existir entre la familia y el centro.

Ante esta realidad, nuestra respuesta supone una doble línea de intervención.

Para el conjunto de nuestro alumnado en general se incluyen en las programaciones de tutoría objetivos y actividades encaminados a desarrollar actitudes de aceptación y apertura hacia la diferencia, y se destinan tiempos y espacios en los que es posible escuchar sus preocupaciones y se les proporcionan, también, explicaciones para mejorar la relación con los compañeros con discapacidad.

Para el grupo de alumnos con N.E.E. introducimos en su currículum, dentro del área afectivo-social, pero con una entidad fundamental y una importancia equiparable a la que tienen las matemáticas o la lengua, el taller de habilidades sociales.

\section{TALLER DE HABILIDADES SOCIALES}

El taller de habilidades sociales se configura como un espacio en el que los alumnos semanalmente pueden expresar sus dificultades de relación, tienen la oportunidad de conocerse mejor y de reflexionar sobre lo que les ocurre y aprenden a tomar decisiones $y$ a resolver sus conflictos.

La problemática que surge y que se trabaja en cada una de las sesiones es muy variada y tiene mucho que ver con lo que sucede en el día a día de las aulas ordinarias: dificultades de comunicación, de comprensión y expresión de las situaciones sociales, problemas de liderazgo, falta de motivación, ganas de pertenecer y sentirse parte de un grupo, miedos, inseguridades, rechazos, etcétera.

Los objetivos que se programan se organizan en torno a los tres ejes expuestos en las etapas anteriores: 
I. Conocimiento de sí mismos en todas sus facetas: características personales, gustos y aficiones, puntos débiles, puntos fuertes, autocontrol, sentimientos, intereses, motivaciones, toma de decisiones, etcétera.

2. Conocimiento del otro y relación con él: resolución de conflictos, respeto, identificación de sentimientos, gustos y opiniones, relaciones afectivosexuales, etcétera.

3. Conocimiento del entorno: desenvolvimiento dentro y fuera del colegio, autonomía urbana, planificación de actividades de ocio, estrategias necesarias para hacer planes, proyecto de vida, etcétera.

La dinámica de trabajo de cada sesión parte de una asamblea como punto de partida en la que los alumnos expresan todo aquello que les interesa o preocupa y a continuación se ponen en práctica toda una serie de técnicas entre las que destacamos:

- Ensayo de la conducta: el alumno identifica el problema que ha tenido y que no ha sabido resolver esa semana. Si hay más partes implicadas y están presentes, se escucha la descripción que cada uno haga del problema en cuestión. A continuación se busca una solución aceptable y se ensaya para poderla poner en práctica cuando el problema vuelva a surgir.

- Modelado y role playing: se buscan distintas soluciones o maneras de actuar y se modelan delante de todos para que los alumnos perciban cómo se puede responder ante una misma situación de distintas formas. En primer lugar se escenifica lo que se ha hecho y no ha servido y, posteriormente, se representan las otras formas de actuar.

- Instrucciones: todas las soluciones se acompañan de explicaciones asequibles a los alumnos con argumentos sobre la manera adecuada de relacionarse con los demás, tanto en el entorno escolar como fuera de él. Es preciso dotar a los alumnos de una base ética y práctica que oriente su comportamiento en situaciones futuras.

- Tareas para casa: en ocasiones se proponen actividades para casa, ya sea, de reflexión o de carácter práctico. En estos casos se trata de que los alumnos impliquen a sus padres y obtengan su colaboración.

- Técnicas cognitivas: encaminadas a modificar ideas irracionales, expectativas irreales, baja autoestima, falsos niveles de atribución, miedos, ideas preconcebidas, etcétera.

- Generalización y transferencia: a través de las tareas para casa o de diversas actividades de ocio que se les proponen, se pretende que pongan en práctica las formas de actuar que han aprendido en condiciones diferentes.

La evaluación del taller de habilidades sociales es trimestral como cualquier otra área del currículum. En cada trimestre se seleccionan los ítems más significativos de los objetivos que se han trabajado, por ejemplo: a) es capaz de poner en palabras sus sentimintos de miedo; y b) es capaz de escuchar y dar consejo cuando alguien lo está pasando mal.

\section{ágora de profesores}

Para facilitar a los maestros de apoyo y demás integrantes del equipo interdisciplinario la selección y diseño de aprendizajes esperados tendientes al desarrollo de habilidades sociales, hemos decidido incorporar el Programa de Habilidades Sociales (PHS) de Miguel Ángel Verdugo Alonso (1997), haciendo algunos ajustes pertinentes para nuestra población.

El programa está conformado por seis objetivos generales:

I. Desarrollo de la comunicación verbal requerida para que el alumno partícipe en conversaciones.

2. Desarrollo de las habilidades de interacción social necesarias para que el alumno llegue a establecer y mantener relaciones interpersonales.

3. Desarrollo de las habilidades sociales "instrumentales" que posibiliten en el alumno un funcionamiento lo más autónomo posible.

4. Adquisición de repertorios de conducta adecuados para que el alumno asista, participe y se integre en actos sociales y recreativos.

5. Adquisición y desarrollo de las conductas necesarias para que el alumno conozca y utilice los servicios de la comunidad.

6. Desarrollo progresivo del sentido cívico y la conciencia ciudadana, de modo que se facilite el bienestar social del alumno y su convivencia con el entorno social y natural.

La calificación tiene tres categorías:

I. Si el ítem está sólo en fase inicial.

2. Si está en fase de trabajo.

3. Si ya se ha conseguido.

Junto con la calificación se suele incluir una nota explicativa de cómo el alumno está asimilando el objetivo en cuestión.

Los criterios para evaluar el grado de consecución de los objetivos programados en el taller de habilidades sociales son los siguientes.

- Autoconocimiento, confianza en sí mismo, aceptación de las circunstancias personales.

- Capacidad para expresar sentimientos y opiniones.

- Capacidad para respetar al otro y poderse poner en el lugar de los demás.

- Capacidad para organizar actividades de ocio y tiempo libre.

- Capacidad para desenvolverse de forma autónoma dentro y fuera del centro.

- Capacidad para resolver conflictos.

- Capacidad para albergar objetivos de mejora y progreso personal y social. 


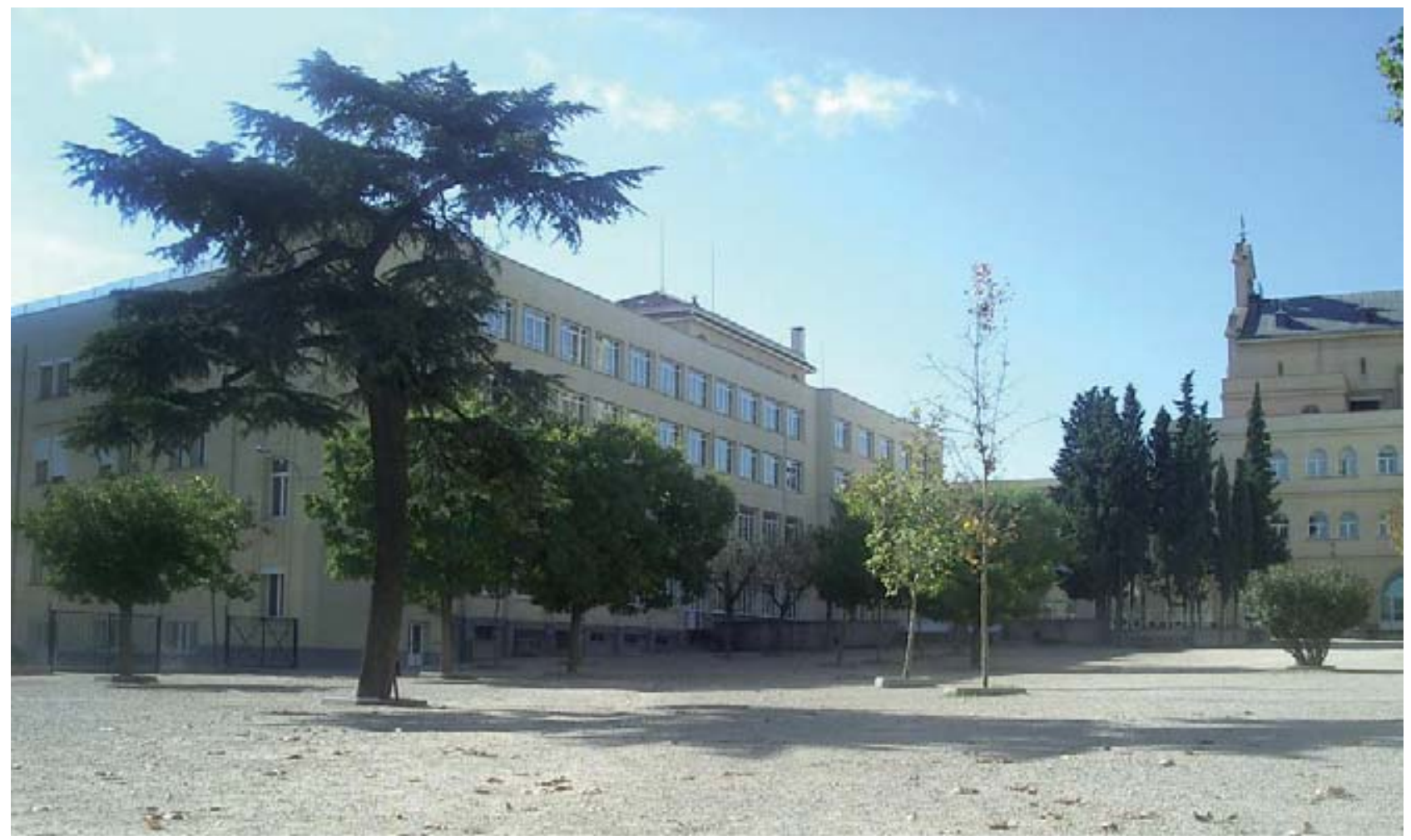

Colegio Sagrado Corazón Chamartín (Madrid).

La experiencia que nos proporcionan las promociones de alumnos con discapacidad que terminan su escolaridad en el colegio nos demuestra que éstas son las capacidades esenciales que hacen posible que estos chicos estén en condiciones de llevar a cabo un proyecto vital autónomo en la medida de sus posibilidades personales.

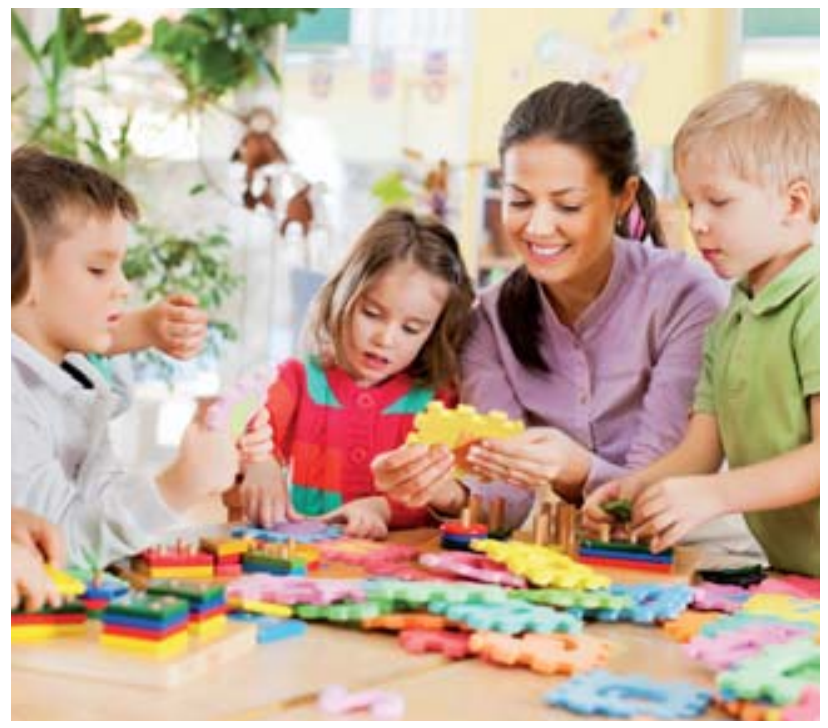

\section{Para saber más}

- Hernández Muñoz, G. (20I2). Saberes y quehaceres de los maestros de apoyo, reflexiones en torno a la discapacidad intelectual y a la escuela inclusiva. México: Instituto de Educación de Aguascalientes, enero.

- IzuzQuiza, D., y Ruiz, R. (2007). Tú y yo aprendemos a relacionarnos. España: Programa para la Enseñanza de Habilidades Sociales en el Hogar.

- Monjas Cásares, M. I. (1999). Programa de enseñanza de habilidades de interacción social para niños y niñas en edad escolar (PEHIS). Madrid.

- Verdugo, M. Á. "Análisis de la definición de la discapacidad intelectual". Siglo Cero. Revista Española sobre Discapacidad Intelectual vol. 34 (I), n.' 205.

- Verdugo Alonso, M. Á., y Gutiérrez Bermejo, B. (2009). Discapacidad Intelectual. Adaptación social y problemas de comportamiento. España: Ediciones Pirámide.

- Veved Conte, E.; Páramo, M. S., y otros (20l0). Programa de Habilidades Sociales, autonomía personal y autorregulación. Aplicación con alumnos con S. de Down. Down España.

- Páginas web: www.centrodocumentacióndown. com 